\title{
Variation annuelle de l'état nutritionnel de la lapine Angora durant les pousses saisonnières des poils
}

\author{
Geneviève CHARLET-LERY (*), Michèle FISZLEWICZ ("), \\ Marie-Thérèse MOREL $(*)$, J. ROUGEOT (**) et R.G. THÉBAULT (**) \\ I.N.R.A., C.N.R.Z. \\ * Laboratoire de Physiologie de la Nutrition \\ *: Laboratoire des Pelages, Toisons et Fourrures \\ F 78350 Jouy-en-Josas
}

\begin{abstract}
Résumé
Très peu d'études nutritionnelles ont été effectuées jusqu'à maintenant sur le lapin Angora, animal épilé 4 fois par an pour la récolte du poil qu'il produit.

Utilisant le plan alimentaire de la lapinerie d'élevage du C.N.R.Z., invariable quelle que soit la saison et régressif avec l'approche de l'épilage (tabl. 2), nous avons, durant 52 semaines, mesuré hebdomadairement le poids de 9 lapines, leur niveau d'ingestion et, au cours des $2^{\prime \prime}, 7^{\prime \prime}$ et $12^{\prime \prime}$ semaines post-épilage, les coefficients de digestibilité et les bilans d'azote $(n=104)$. Les épilages ont été effectués toutes les 13 semaines.

L'évolution pondérale n'est pas ou peu influencée par la saison. Elle est influencée par le rythme des épilages : diminution du poids vif «nu» au cours des jours qui le précèdent (ralentissement de l'ingéré et régression de la cæcotrophie) et dans les jours qui le suivent (modification brutale de l'environnement surtout en saisons froides), puis reprise progressive (fig. 1 et 2 ).

Malgré des ingestions moyennes très homogènes, les bilans $\mathrm{N}$ et les $\mathrm{CR}$ sont statistiquement plus faibles en automne et plus élevés en hiver (tabl. 7).

La diminution de l'ingestion entre les $2^{\prime \prime}, 7^{\circ}$ et $12^{\prime \prime}$ semaines ne modifie pas les CUD MO ; les CUD N sont plus laables in 12" semaine par suite de la présence, avec les crottes dures, de cæcotrophes trois fois plus riches en $\mathrm{N}$ que les premières (tabl. 5). Le rendement annuel du dépôt protéique de poil serait de 18,5 p. 100.

On peut penser que la modification brutale de l'environnement thermique provoquée par l'épilage, serait la cause des pertes pondérales et sans doute de la mortalité maximale à ce moment dans les élevages.
\end{abstract}

Mots clés : Lapin angora, saison, production de poils.

\section{Introduction}

Le lapin Angora est élevé uniquement pour la production de poil qui atteint dans les meilleurs élevages près de $1 \mathrm{~kg}$ par an. 
En France, le poil Angora est récolté par épilation. L'opération est en fait grandement facilitée par la maturité du pelage qui correspond à un taux déjà élevé de poils prêts à tomber. Cependant, malgré cet état de maturité, l'épilation provoque chez l'animal un stress plus ou moins accentué ; en outre, le lapin reste dénudé pendant 2 semaines. A ces cycles trimestriels très marqués de la couverture pileuse, s'ajoutent des variations saisonnières de la production poilière qui se manifestent notamment par un creux estival qui peut parfois atteindre 20 p. 100 en moins par rapport au poids de la toison d'hiver (Rougeot \& ThÉbault, 1983).

Il en résulte que les besoins nutritionnels, tant énergétiques que protéiques, dépendent des cycles de production poilière et du pouvoir isolant de la toison. Or le lapin autorégule mal sa consommation (Rougeot \& Thébault, 1977 ; Scholaut \& LANGE, 1983). On ne peut donc distribuer au lapin Angora les aliments concentrés, céréales ou agglomérés concentrés ad libitum, sans risque d'accident. Ce sont en effet des adultes dont l'état physiologique est celui de l'entretien, avec par conséquent des capacités digestives limitées et auxquels on demande de réaliser un potentiel de production de poils que la sélection ne cesse d'élever. D'autre part, l'ingestion au cours de la toilette de longs poils qui, après 2 mois et demi de repousse, ne tiennent pas tous solidement à la peau, provoque, lorsque l'estomac est encombré, la formation de trichobézoards : ce risque est d'autant plus élevé que l'ingestion des aliments concentrés est rapide et abondante (Rougeot \& THÉbaulT, 1977).

Il est donc nécessaire de connaître avec précision les besoins nutritionnels du lapin Angora, afin d'établir un plan de rationnement qui, tout en permettant de fournir la production maximale de poil, offre toute sécurité pour la santé et la survie de l'animal.

Ainsi, nous proposons-nous dans cette étude de mesurer, chez la lapine Angora adulte qui fournit quelques 20 p. 100 de plus de poils Angora que le mâle, comment varient, au cours d'une année, le niveau d'ingestion, les coefficients de digestibilité et de rétention azotée et le poids de l'animal en fonction du stade de croissance de la toison au cours de chacune des 4 productions saisonnières.

\section{Matériel et méthodes}

\section{A. Animaux}

Neuf lapines Angora adultes, issues de l'élevage expérimental de l'I.N.R.A., ont été synchronisées pour les dates de récolte du poil de façon à pouvoir être épilées sur deux jours au maximum. Le poids moyen des animaux, pris immédiatement après l'épilation marquant le début de l'expérience, le 17 mars 1982, s'élevait à $3813 \pm$ $69 \mathrm{~g}$. Ces lapines ont été maintenues pendant un an, jusqu'au 17 mars 1983, date de la $4^{\prime \prime}$ et dernière épilation de l'expérience, dans une salle expérimentale close, éclairée par la lumière du jour passant par une baie vitrée orientée à l'est. Elles sont donc restées sous l'influence du photopériodisme naturel régnant à $45^{\circ}$ de latitude Nord. La température y a varié de +14 à $+24{ }^{\circ} \mathrm{C}$ selon les saisons. 


\section{B. Cages à bilan et modalités expérimentales}

Nous avons logé en permanence les lapines Angora dans des cages à bilans en grillage de $52 \times 52 \mathrm{~cm}$. Hors des périodes de bilans, le plancher grillagé est recouvert d'une plaque de fibro-ciment, percée de quelques trous pour assurer l'écoulement de l'urine et qui retient la litière de paille indispensable au confort et au maintien en bon état physiologique des animaux. Les lapines ingèrent évidemment une partie de cette paille : c'est pourquoi elle est supprimée dès le samedi qui précède la période de bilan, s'étendant toujours sur 4 jours, du lundi matin au vendredi matin. La collecte, la pesée et l'échantillonnage des fèces et de l'urine sont effectués chaque jour pendant les périodes de bilan. Les échantillons sont aussitôt conservés à $+5^{\circ} \mathrm{C}$ en vue de leur analyse.

Au cours des bilans, les contrôles des refus sont effectués tous les jours, mais ces refus sont remis dans les mangeoires et enlevés définitivement le vendredi matin. Un plateau en grillage très fin est placé sous la mangeoire pour collecter les gaspillages. La pesée des animaux est effectuée chaque vendredi afin de la faire coïncider avec la fin des bilans. L'eau de boisson est offerte ad libitum.

Les animaux ont été épilés au cours de la semaine qui précède le changement de la saison $\left(3^{\circ}\right.$ semaine de mars, juin, septembre et décembre), c'est-à-dire tous les 3 mois (13 semaines) afin de faire coïncider parfaitement les cycles de pousse du poil et les saisons. Les bilans ont eu lieu au cours des $2^{\circ}, 7^{\circ}$ et $12^{\circ}$ semaines qui ont suivi chaque épilation.

\section{Régime alimentaire}

La composition des granulés est indiquée au tableau 1. L'apport de cellulose, nécessaire pour le lapin, est obtenu par l'incorporation de paille de blé $(15,7 \mathrm{p} .100$ MS). Selon Rougeot et al. (1980), une telle proportion satisfait les besoins en cellulose du lapin Angora. Les teneurs en protéines brutes et en matières grasses atteignent respectivement 17,8 et 3,1 p. $100 \mathrm{MS}$. L'apport calorique est de $4,8 \mathrm{kcal} / \mathrm{g} \mathrm{MS}$.

Les lapines ont reçu leur ration journalière selon le plan alimentaire en usage à la lapinerie, quelle que soit la saison (tabl. 2). Les quantités distribuées au cours de chaque saison (13 semaines) sont uniformément de 14,4 kg (12,9 kg MS). Entre deux épilages, la ration hebdomadaire est régulièrement diminuée : 1200,1100 et $1000 \mathrm{~g}$ par semaine de la $1^{\text {re }}$ à la $5^{\circ}$, de la $6^{\circ}$ à la $9^{\prime \prime}$ et de la $10^{\circ}$ à la $13^{\circ}$ semaine. Les animaux sont mis à jeun un jour par semaine, le samedi ; ils ont donc reçu, selon les bilans, $189,175,5$ et $157,5 \mathrm{~g}$ de matière sèche par jour en $2^{\circ}, 7^{\circ}$ et $12^{\circ}$ semaine respectivement.

Les bilans durent 4 jours ; ils n'incluent pas le jour de jeûne. Ils sont comparables entre eux, d'une saison à l'autre et entre animaux.

\section{Récoltes des toisons}

La récolte des poils a été effectuée par épilation. Celle-ci a été rendue plus aisée et rapide par l'administration d'un fourrage dépilatoire donné 6 jours auparavant à la place de la ration alimentaire habituelle (Lagodendron de la Société Proval, 


\section{TABLEAU 1}

Composition et analyse chimique du régime utilisé.

Composition and chemical analyses of experimental diet.

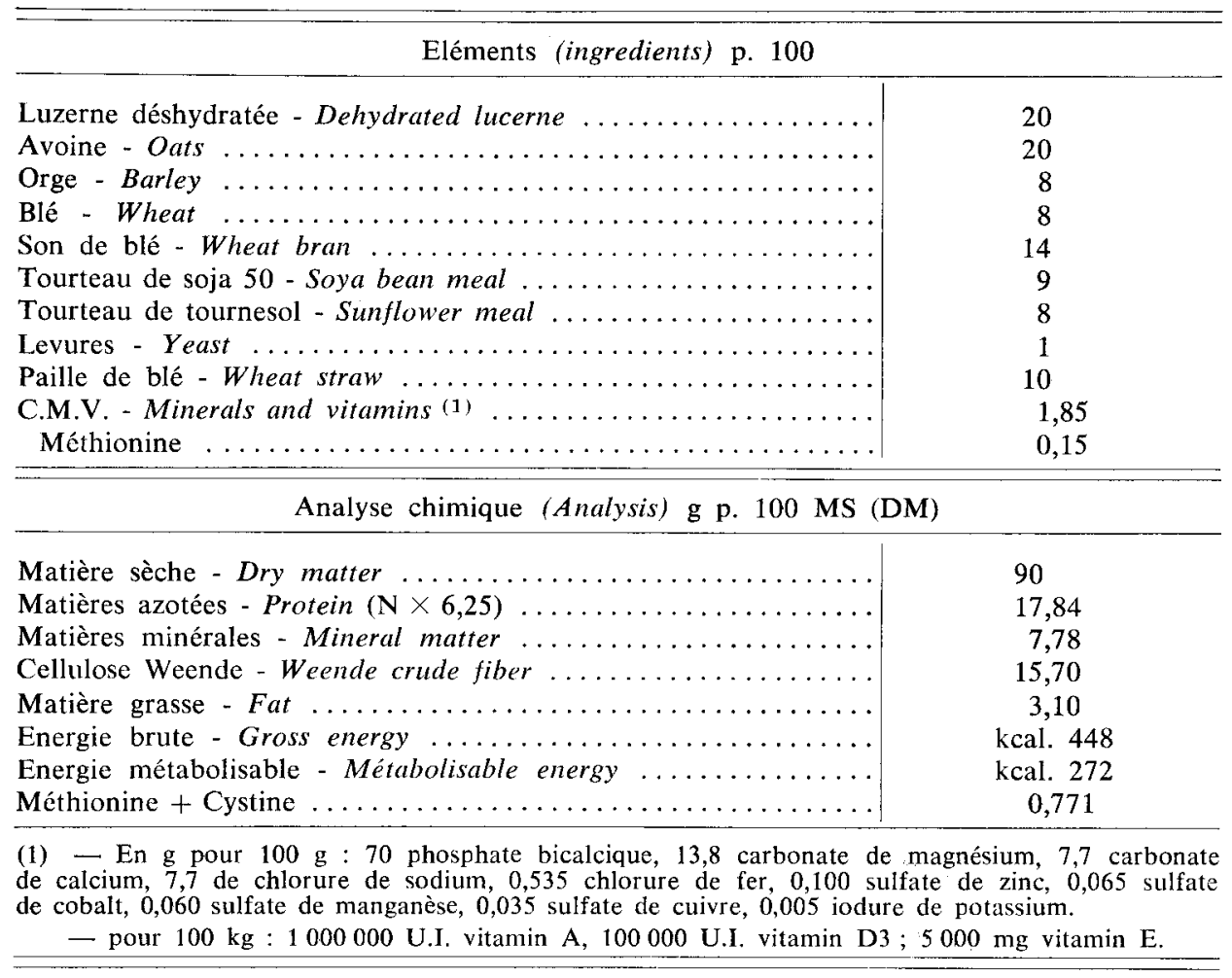

\section{TABleau 2}

Ration alimentaire durant les 13 semaines entre 2 épilages.

Feed distribution during the 13 weeks between 2 depilations.

$\mathrm{g}$

\begin{tabular}{c|c|c|c|c}
\hline \hline $\begin{array}{c}\text { Semaines } \\
\text { Weeks }\end{array}$ & $\begin{array}{c}\text { Ration } \\
\text { hebdomadaire } \\
\text { Weekly feed } \\
\mathrm{g}\end{array}$ & $\begin{array}{c}\text { Dimanche } \\
\text { à jeudi } \\
\text { Sunday } \\
\text { to Thursday } \\
\mathrm{g} / \mathrm{j}\end{array}$ & $\begin{array}{c}\text { Vendredi } \\
\text { Friday } \\
\mathrm{g} / \mathrm{j}\end{array}$ & $\begin{array}{c}\text { Samedi } \\
\text { Saturday }\end{array}$ \\
\hline $\begin{array}{c}1-5^{\circ} \text { sem. - Week ... } \\
6-9^{\mathrm{e}} \text { sem. - Week ... }\end{array}$ & 1200 & 210 & 150 & \\
$10-13^{\circ}$ sem. - Week ... & 1100 & 195 & 125 & $\begin{array}{c}\text { jeûne } \\
\text { jeûne } \\
\text { jeûne }\end{array}$ \\
\hline \hline
\end{tabular}


Paris). Ce traitement n'a aucune influence sur la production de poil, mais atténue les effets du choc épilatoire chez les animaux (Rougeot \& ThÉbaulT, 1985). On mesure le poids des poils recueillis et leur répartition relative selon les diverses qualités, $1^{\text {er }}$ choix $\mathrm{A}$ et $\mathrm{B}, 2^{\mathrm{C}}$ choix poils courts, feutre propre, poil sale.

Les animaux sont pesés avant et après la récolte et les deux jours suivants.

\section{E. Analyses chimiques}

La concentration énergétique est mesurée avec une bombe adiabatique Gallemkamp. La matière organique est calculée par différence entre la matière sèche et la matière minérale. L'azote est dosé par la méthode de Kjeldahl.

Les résultats sont donnés avec leur erreur standard.

\section{Résultats}

\section{A. Production de poil}

La production moyenne de poil des 9 lapines étudiées a atteint pendant l'année de l'expérience, $983 \pm 31 \mathrm{~g}$, variant suivant les animaux de 841 à $1132 \mathrm{~g}$ (tabl, 3).

\section{TABLeaU 3}

Ingéré brut et production de poils.

Feed intake and hair production.

Animal/saison $\pm \mathrm{Sm}(\mathrm{n}=9)$

Animal/season

\begin{tabular}{|c|c|c|c|c|c|}
\hline $\begin{array}{l}\text { Saison } \\
\text { Season }\end{array}$ & $\begin{array}{l}\text { Printemps } \\
\text { Spring }\end{array}$ & $\begin{array}{c}\text { Eté } \\
\text { Summer }\end{array}$ & $\begin{array}{l}\text { Automne } \\
\text { Autumn }\end{array}$ & $\begin{array}{l}\text { Hiver } \\
\text { Winter }\end{array}$ & Total \\
\hline $\begin{array}{l}\text { Ingéré }(\mathrm{kg}) \ldots . . . . . \\
\text { Feed intake } \\
\text { (kg) }\end{array}$ & $\begin{array}{r}14,07 \\
\pm 0,09\end{array}$ & $\begin{array}{r}13,41 \\
\pm 0,20\end{array}$ & $\begin{array}{r}13,64 \\
\pm 0,16\end{array}$ & $\begin{array}{r}13,63 \\
\pm 0,21\end{array}$ & $\begin{array}{r}54,75 \\
\pm \quad 0,57\end{array}$ \\
\hline $\begin{array}{l}\text { Poils }(g) \\
\text { Hair }(g)\end{array}$ & $\begin{array}{l}247,1 \\
\pm 8,2\end{array}$ & $\begin{array}{l}231,7 \\
\pm 9,0\end{array}$ & $\begin{array}{l}249,2 \\
\pm 8,6\end{array}$ & $\begin{array}{l}255,3 \\
\pm 9,5\end{array}$ & $\begin{array}{r}983 \\
\pm 31\end{array}$ \\
\hline r $\ldots \ldots \ldots$ & $+0,30$ & 0,00 & $+0,05$ & $+0,13$ & $+\quad 0,010$ \\
\hline
\end{tabular}

Le même tableau indique simultanément la moyenne des productions individuelles par saison. Le minimum est observé en été : $231,7 \pm 9,0 \mathrm{~g}$, le maximum en hiver : $255,3 \pm 9,5 \mathrm{~g}$; les deux autres saisons sont intermédiaires. Seuls 3 animaux ont des variations importantes entre leurs productions saisonnières extrêmes, l'un 41 p. 100, deux autres 21 p. 100. Par contre, il est intéressant de remarquer que les 2 animaux 
dont les productions poilières ont atteint ou dépassé $1,1 \mathrm{~kg}$ présentent une amplitude de variation saisonnière faible, voisine de 5 p. 100 ; toutefois, il n'y a pas de relation significative entre cette amplitude et la production annuelle.

\section{B. Niveau d'ingestion}

Le tableau 3 indique les quantités moyennes d'aliments ingérés au cours des diverses saisons. L'ingéré minimum est observé en été : $13,41 \mathrm{~kg} \pm 0,20$; il est très proche des ingérés d'automne et d'hiver : $13,64 \mathrm{~kg} \pm 0,16$ et $13,63 \mathrm{~kg} \pm 0,21$, mais se différencie de la donnée observée au printemps : 14,07 kg $\pm 0,09 \mathrm{~kg}$.

L'ingéré moyen, sur l'année, atteint $54,75 \pm 0,57 \mathrm{~kg}$. Par rapport aux quantités distribuées, $14,4 \mathrm{~kg}$ par saison et $57,6 \mathrm{~kg}$ durant les 52 semaines, les ingérés représentent $97,7,93,1,94,7$ et 94,7 p. 100 du distribué pour les 4 saisons successives et 95 p. 100 pour l'ensemble des données.

La production de poil semble indépendante du niveau d'ingestion $(\mathrm{r}=+0,01)$, mais ce dernier ne varie que de 10 p. 100 entre les animaux extrêmes $(51,46$ à $57,47 \mathrm{~kg} / \mathrm{an})$.

\section{Evolution pondérale}

Les pesées hebdomadaires permettent de connaître l'évolution du poids des lapines. Il est évident que, de ce poids global, doit être déduit le poids des poils que l'animal porte et qui dépasse, à l'approche de la récolte, largement 200 grammes. La connaissance de l'évolution de la production de poil semaine par semaine (Rovgeot \& ThéBault, 1983), et du poil récolté à chaque épilage pour chacun des animaux, permet l'estimation de la production journalière et, par différence, le poids de l'animal nu, à chaque pesée. La figure 1 montre l'évolution pondérale moyenne, poils inclus et poils exclus, des 9 lapines au cours d'une saison (la saison hivernale); la figure 2 montre leur évolution pondérale, hors poil, durant les 4 saisons consécutives. L'examen de ces courbes entraîne les remarques suivantes :

- l'évolution entre deux récoltes de poil est sensiblement identique quelle que soit la saison;

- au cours des semaines qui précèdent l'épilage, les lapines ont tendance à maigrir légèrement;

- l'épilage lui-même, dont la durée a varié de 15 à 30 minutes, provoque une chute de poids «nu» de l'ordre de $10 \mathrm{~g}$, expliquée essentiellement par des émissions fécales ou urinaires. Par contre, les pesées effectuées le lendemain $(E+1)$ mettent en évidence des chutes de poids importantes : - 141. g par animal (de 70 à $220 \mathrm{~g}$ ) qui s'atténuent dans les jours suivants : $-132 \mathrm{~g}$ à $\mathrm{E}+2,-103 \mathrm{~g}$ à $\mathrm{E}+3$. Toutefois, 9 ou 10 jours après l'épilage, les différences pondérales par rapport au "poids nu » juste avant épilage, ne sont pas encore totalement résorbées $(-40 \mathrm{~g}$ en été, $-81 \mathrm{~g}$ en automne, $-138 \mathrm{~g}$ en hiver et $-116 \mathrm{~g}$ au printemps) (tabl. 4). Il faut attendre environ 3 semaines pour retrouver le poids initial ;

- la comparaison des «poids nus » juste après épilation, au début des printemps 1982 et $1983,3813 \pm 69 \mathrm{~g}$ et $4018 \pm 114 \mathrm{~g}$ respectivement, en 52 semaines exactement, met en évidence un gain pondéral moyen de $205 \mathrm{~g}$. Mais les 9 lapines se séparent en 2 groupes : quatre d'entre elles maigrissent ou gagnent peu de poids, entre $-258 \mathrm{~g}$ et $+86 \mathrm{~g}$, cinq grossissent notablement entre 200 et 680 grammes. 


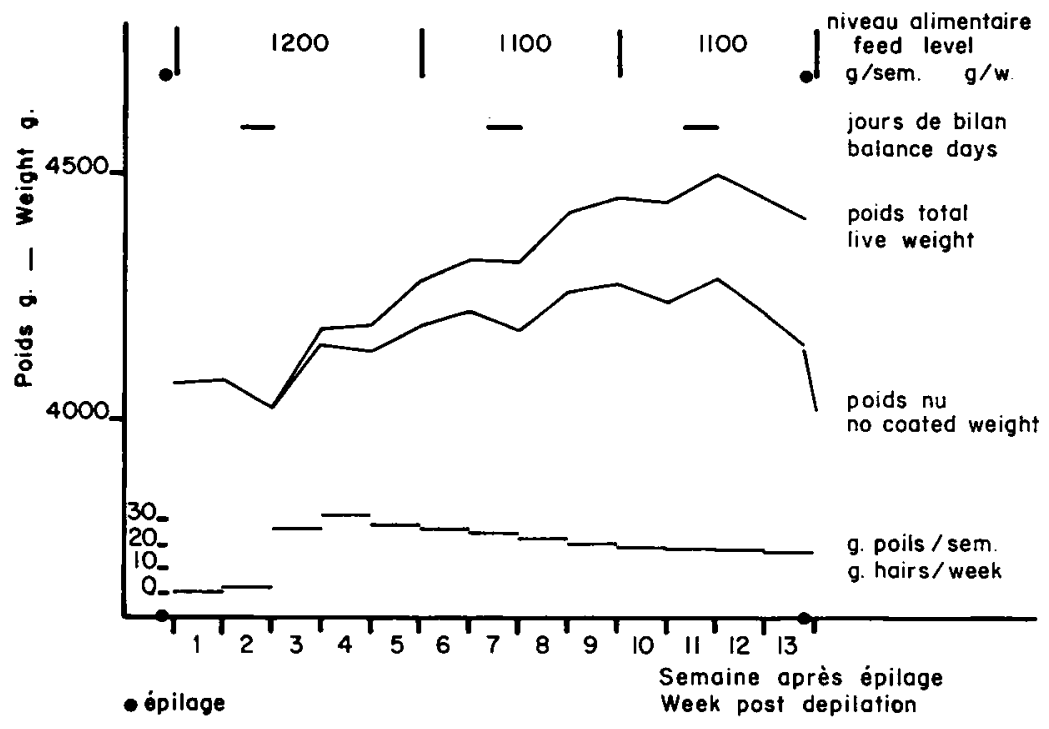

FIG. 1

Variation pondérale (totale et poids nu) et production de poil au cours de l'hiver.

Live weight variation (total and no coated) and hair production during winter.

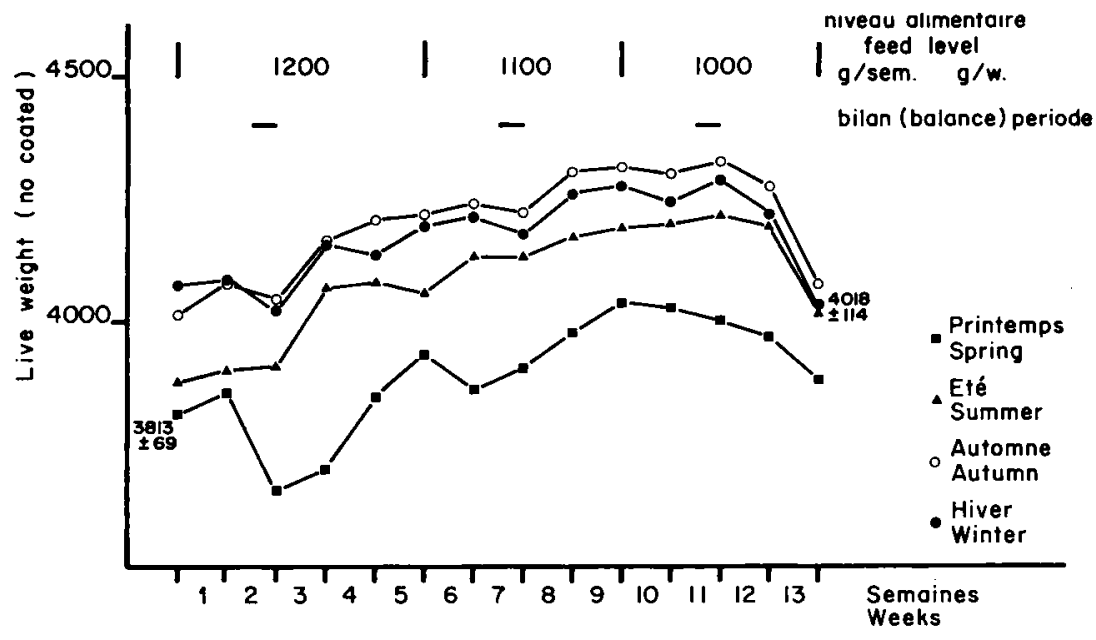

FIG. 2

Variation du poids nu entre chaque épilage $(n=9)$.

Variation of no coated weight between two depilations $(n=9)$. 


\section{TABLEaU 4}

Perte de «poids nu» consécutive à l'épilage selon les saisons.

Weight losses induced by depilation according to the season.

$\mathrm{g}$

\begin{tabular}{|c|c|c|}
\hline $\begin{array}{c}\text { Nombre de jours après l'épilation } \\
\text { Days after depilation }\end{array}$ & 1 & $8-9$ \\
\hline Printemps (mars) - Spring (March) & -147 & -116 \\
\hline Eté (juin) - Summer (June) & -128 & -40 \\
\hline Automne (septembre) - Autumn (September) & -139 & -81 \\
\hline Hiver (décembre) - Winter (December) & -151 & -138 \\
\hline
\end{tabular}

Si l'on compare, pour l'ensemble de l'année, les données obtenues entre les deux épilages de printemps, relatives aux quantités de poils produits, à l'ingéré et aux variations de poids (tabl. 5), il existe une corrélation positive entre l'ingéré annuel et la variation de poids $(\mathrm{r}=+0,46)$ et une corrélation négative entre la production de poils et la variation de poids $(r=-0,41)$, ce qui explique l'absence de corrélation déjà évoquée entre l'ingéré et la production de poils $(r=-0,10)$. Aucun de ces coefficients n'est significatif.

\section{TaBleau 5}

Variations pondérales, poids de poils produits et matières sèches ingérées.

Live weight variation, hair production and dry matter ingested.

An - Year

\begin{tabular}{|c|c|c|c|}
\hline $\begin{array}{l}\text { Nombre d'animaux } \\
\text { Number of animals }\end{array}$ & $\begin{array}{l}\text { Variation de poids } \\
\text { des animaux } \\
\text { Live weight variation } \\
\mathrm{g}\end{array}$ & $\begin{array}{l}\text { Production de poils } \\
\text { Hair production } \\
\mathrm{g}\end{array}$ & $\begin{array}{c}\text { Ingéré } \\
\text { Intake } \\
\mathrm{kg} \text { MS (DM) }\end{array}$ \\
\hline $8^{(1)}$ & 248 & 988 & 54,9 \\
\hline $\begin{array}{l}\text { Coefficients } \\
\text { de corrélation }\end{array}$ & $\leftarrow \longleftarrow-0,41-$ & 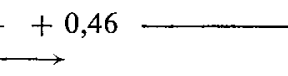 & \\
\hline $\begin{array}{l}\text { Animaux ayant maigri } \\
\text { ou peu grossi } \\
\text { Weight loss or small } \\
\text { weight gain } \\
4\end{array}$ & $\begin{array}{l}-84,5 \mathrm{~g} \\
(-258,+86)\end{array}$ & $\begin{array}{l}1036 \mathrm{~g} \\
(921,1132)(2)\end{array}$ & 53,94 \\
\hline $\begin{array}{l}\text { Animaux ayant grossi } \\
\text { Weight gain } \\
4\end{array}$ & $\begin{array}{l}+412 \mathrm{~g} \\
(+250,+680)(2)\end{array}$ & $\begin{array}{l}941 \mathrm{~g} \\
(841,1030)(2)\end{array}$ & 55,80 \\
\hline \multicolumn{4}{|c|}{$\begin{array}{l}\text { (1) Une lapine est morte quelques jours avant la pesée finale. } \\
\text { A doe-rabbit died some days before final weight. }\end{array}$} \\
\hline \multicolumn{4}{|l|}{$\begin{array}{l}\text { (2) Valeurs extrêmes. } \\
\text { Extrome values. }\end{array}$} \\
\hline
\end{tabular}




\section{Bilans}

Sur les 108 bilans individuels (9 animaux, 12 bilans), quatre d'entre eux ont dû être supprimés au cours des $1^{\mathrm{re}}$ (animal 3), des $3^{\circ}$ et $6^{\circ}$ (animal 9) et $12^{\circ}$ (animal 1) périodes; mais sauf pour l'animal 9 , malade, les coefficients de digestibilité ont pu être utilisés. De plus, lors du premier bilan, nous avons constaté des blessures aux pattes arrière des animaux par suite du contact direct sur le grillage. Ce bilan a été limité à 3 jours. Ultérieurement, les pattes ont été protégées.

Au cours des deux premiers bilans effectués $\left(2^{\circ}\right.$ et $7^{\circ}$ semaines post-épilatoire de printemps), la comparaison entre les coefficients d'utilisation digestive de l'énergie CUD E et ceux de la matière organique CUD MO a mis en évidence l'égalité des 2 séries de données (tabl. 6). Ce résultat nous permet de n'utiliser que le second de ces coefficients.

Le tableau 7 regroupe l'ensemble des données obtenues par saison : printemps, été, automne, hiver, et le tableau 8 l'ensemble des données obtenues selon les semaines post-épilage : $2^{\mathrm{e}}, 7^{\mathrm{e}}$ et $12^{\mathrm{e}}$ semaine.

\section{TABLEAU 6}

Comparaison entre les coefficients d'utilisation digestive de la matière organique et de l'énergie $(n=9)$.

CUD organic matter in comparison with CUD energy $(n=9)$.

$$
\mathrm{x} \pm \mathrm{Sm}
$$

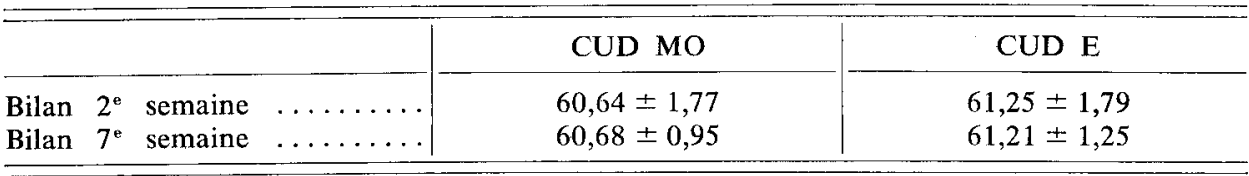

\section{Tableau 7}

Variations saisonnières.

Seasonal variations.

Moyenne des 3 bilans.

Average of the $3 N$-balances.

$$
\pm \mathrm{Sm}
$$

\begin{tabular}{l|c|c|c|c}
\hline & $\begin{array}{c}\text { Printemps } \\
\text { Spring } \\
\mathrm{n}=25(1)\end{array}$ & $\begin{array}{c}\text { Eté } \\
\text { Summer } \\
\mathrm{n}=26(1)\end{array}$ & $\begin{array}{c}\text { Automne } \\
\text { Autumn } \\
\mathrm{n}=27\end{array}$ & $\begin{array}{c}\text { Hiver } \\
\text { Winter } \\
\mathrm{n}=26(1)\end{array}$ \\
\hline MSI $(\mathrm{g} / \mathrm{j}) \ldots \ldots$ & $161,6 \pm 5,4$ & $162,4 \pm 4,5$ & $165,8 \pm 3,4$ & $165,8 \pm 4,0$ \\
NI $(\mathrm{g} / \mathrm{j}) \ldots \ldots$ & 4,65 & 4,66 & 4,79 & 4,68 \\
CUD MO ... & 60,3 & 59,6 & 60,1 & 60,6 \\
CUD N . . . & 78,2 & 75,4 & 75,0 & 78,9 \\
Bilan N $(\mathrm{g} / \mathrm{j}) \ldots$ & $1,19 \pm 0,08 \mathrm{ab}$ & $1,12 \pm 0,07 \mathrm{ab}$ & $1,06 \pm 0,07 \mathrm{a}$ & $1,22 \pm 0,06 \mathrm{~b}$ \\
CR ....... & $33,2 \pm 1,5 \mathrm{~b}$ & $32,5 \pm 0,9 \mathrm{~b}$ & $28,9 \pm 1,3 \mathrm{a}$ & $34,1 \pm 1,2 \mathrm{bc}$ \\
\hline
\end{tabular}

(1) Suppression des données relatives aux bilans $N$ pour la lapine 3 , durant le $1^{\text {er }}$ bilan $(0,30)$ pour la lapine 9 durant les $3^{\mathrm{e}}$ et $6^{\mathrm{e}}$ bilans $(0,01$ et 0,13$)$, anormalement faibles par rapport à l'ensemble des autres données et pour la lapine 1 malade au cours du $10^{\circ}$ bilan.

Compared to average values, N-retention was very low in doe-rabbit 3 during the 1 st period $(0.30 \mathrm{~g} / \mathrm{d})$ and doe-rabbit 9 during periods 3 and $6(0.01-0.13)$; doe-rabbit 1 was ill during period 10. They were discarded. 


\section{Niveau alimentaire}

Comme pour l'ingéré global, la moyenne des ingérés des 3 bilans effectués par saison varie très peu.

Au cours des différents bilans d'une même saison, l'ingéré est en accord avec le plan alimentaire, les refus ayant été faibles, 2 p. 100 en $2^{\circ}$ semaine, 5 et 11 p. 100 en $7^{\circ}$ et $12^{\mathrm{e}}$ semaine. Au cours des 12 semaines, un seul animal a toujours ingéré la totalité de sa ration : tous les autres ont laissé, au moins une fois, un refus journalier supérieur à 10 p. $100 \mathrm{du}$ distribué.

\section{Coefficient de digestibilité}

Comme pour les ingérés, les variations des coefficients de digestibilité, en fonction des saisons, sont faibles et non significatives.

Les $2^{\mathrm{e}}$ et $7^{\mathfrak{b}}$ semaines conduisent à des résultats très proches, tandis qu'en $12^{\circ}$ semaine les données obtenues sont nettement inférieures. Les CUD MO sont relativement faibles et peu variables, mais la différence entre la $2^{\circ}(61,1 \pm 0,3)$ et la $12^{\circ}$ semaine $(59,1 \pm 0,7)$ est significative $(P<0,01)$. Cette différence devient hautement significative lorsque l'on considère les données relatives à l'azote : 78,6 $\pm 0,5$ et $72,1 \pm 1,5$, respectivement en $2^{\circ}$ et $12^{\circ}$ semaine. En $7^{\circ}$ semaine, les différences avec la $2^{\mathrm{e}}$ semaine sont faibles et irrégulières, légèrement inférieures ou supérieures : $77,5 \pm 0,9$; cette valeur est significativement différente de celle observée en $12^{\circ}$ semaine $(\mathrm{P}<0,01)$.

La raison évidente de cette chute est la différence des teneurs en azote des fèces au cours du cycle saisonnier : $1,478 \pm 0,030,1,560 \pm 0,031$ et $1,856 \pm 0,035$ respectivement pour les $2^{\circ}, 7^{*}$ et $12^{\circ}$ semaines après épilage (tabl. 8). Deux phénomènes peuvent expliquer la plus forte concentration en $12^{\mathrm{e}}$ semaine :

1) la présence de poils inclus dans la masse fécale, poils qui lient souvent les crottes entre elles, formant des chapelets (Rougeot \& ThÉbault, 1977). La quantification de ces derniers a été faite consécutivement à cette expérience sur un autre groupe de lapines par immersion et désagrégation des crottes dans l'eau, séparation des poils et séchage à l'étuve. En 4 jours de bilans, il a été récolté $0,34 \mathrm{~g}$ de poil par jour, ce qui représente, si l'on tient compte d'une teneur en protéines de 85 p. 100, un supplément de $0,046 \mathrm{~g} \mathrm{~N}$ dans la masse fécale journalière. En déduisant cet apport «extérieur», les CUD $\mathrm{N}$ sont augmentés de 1 point, passant de 71,1 à 72,1 . Ce sont les données corrigées qui ont toujours été prises en considération ;

2) la présence, dès la $11^{\circ}$ semaine, d'une certaine quantité de cæcotrophes aisément visibles par suite de leur teinte, de leur taille (plus petite) et de leur luisance, cæcotrophes plus riches en azote et en eau que les crottes dures (DEmaux et al., 1980).

La connaissance de la concentration en matière sèche des crottes dures : 73,11 $\pm 0,75$, moyenne de nos résultats enregistrés en $2^{\mathrm{e}}$ semaine, proche de la donnée de Demaux et al. : 74,2 $\pm 1,3$ et de celle des crottes molles : 41,9 $\pm 1,4$ indiquée par les mêmes auteurs, permet de calculer pour la $12^{\circ}$ semaine, durant laquelle la teneur en matière sèche est de $70,42 \pm 0,69$, les quantités relatives de matières sèches en provenance des crottes dures et des cæcotrophes : 90,8 et 9,2 p. 100 soit en brut 84,9 et 15,1 p. 100 . 


\section{TABleau 8}

Ingéré, coefficients de digestibilité et retention azotée au cours des $2^{\prime \prime}, 7^{\circ}$ et $12^{\circ}$ semaines post épilage.

Intake, nutrien digestibility and $N$ retention during 2 nd, 7 th and 12 th weeks post depilation.

9 animals $\quad \mathrm{x} \pm \mathrm{Sm}$

\begin{tabular}{|c|c|c|c|}
\hline $\begin{array}{l}\text { Semaine postépilage } \\
\text { Post depilation week }\end{array}$ & $\begin{array}{l}2^{r} \text { semaine } \\
2 n d \text { week } \\
(\mathrm{n}=34)\end{array}$ & $\begin{array}{c}7^{\circ} \text { semaine } \\
7 t h \text { week } \\
(\mathrm{n}=35)\end{array}$ & $\begin{array}{c}12^{\mathrm{e}} \text { semaine } \\
12 t h \text { week } \\
(\mathrm{n}=35)(1)\end{array}$ \\
\hline $\begin{array}{l}\text { Matière sèche ingérée }(\mathrm{g} / \mathrm{j}) \ldots \\
\text { Dry matter intake }\end{array}$ & 185,5 & 166,3 & 140,9 \\
\hline $\begin{array}{l}\text { Ingéré p. } 100 \text { distribué } \ldots \ldots \\
\text { Ingested p. } 100 \text { distributed }\end{array}$ & 98,0 & 94,5 & 89,0 \\
\hline $\begin{array}{l}N \text { ingéré }(g / \mathrm{j}) \\
N \text { intake }(g / d)\end{array}$ & $5.28 \pm 0,11 \mathrm{a}$ & $4,81 \pm 0,28 a b$ & $3,98 \pm 0,34 b$ \\
\hline $\begin{array}{l}\text { CUD MO p. } 100 \ldots \ldots \ldots \\
\text { OM digestibility }\end{array}$ & $61,1 \pm 0,30$ & $60,5 \pm 0,6$ & $59,1 \pm 0,7$ \\
\hline $\begin{array}{l}\text { CUD } \mathrm{N} \text { p. } 100 \\
N \text { digestibility }\end{array}$ & $78,6 \pm 0,5 b$ & $77,5 \pm 0,9 b$ & $72,1 \pm 1,5 a$ \\
\hline $\begin{array}{l}\text { Bilan } \mathrm{N}(\mathrm{g} / \mathrm{j}) \ldots \ldots \ldots \ldots \ldots \\
N \text { balance }(g / d)\end{array}$ & $1,30 \pm 0,04 b$ & $1,36 \pm 0,04 b$ & $0,79 \pm 0,05 \mathrm{a}$ \\
\hline $\begin{array}{l}\text { Coefficient de retention } \ldots \ldots \\
N \text { retention coefficient }\end{array}$ & $31.2 \pm 1,0$ & $36,1 \pm 0,9$ & $28,6 \pm 0,2$ \\
\hline $\begin{array}{l}\text { Composition chimique des ma- } \\
\text { tièrcs fécales } \\
\text { Chemical composition of feces } \\
\text { MS }(D M) \text { p. } 100 \ldots \ldots \ldots \ldots \\
\text { N p. } 100 \text { MS }(D M) \ldots \ldots \ldots\end{array}$ & $\begin{array}{c}73,6 \\
1,478 \pm 0,030\end{array}$ & $\begin{array}{c}69,2 \\
1,560 \pm 0.031\end{array}$ & $\begin{array}{c}70,6 \\
1,856 \pm 0,035\end{array}$ \\
\hline
\end{tabular}

(1) Voir tab. 7 - See table 7 .

\section{Bilan azoté}

Rappelons que les bilans d'azote ont été effectués durant 4 jours, au moment où, sclon le plan hebdomadaire utilisé, les animaux ingèrent le plus (tabl. 2). Leurs valcurs sont donc supérieures à celles qui auraient été obtenues si les mesures avaient été effectuées durant 7 jours puisque les ingérés moyens journaliers de 7 jours ne représentent, selon les semaines, que $81,65,80,57$ et 81,65 p. 100 des ingérés moyens de 4 jours.

Au cours des différentes saisons, l'homogénéité des ingérés et le peu de différence entre les coefficients de digestibilité et les productions individuelles de poils sont à relier à la faible amplitude de variations des bilans d'azote moyens. Seuls sont signi- 
ficativement différents l'un de l'autre $(P<0,01)$ les bilans automne $(1,06 \pm 0,07)$ et hiver $(1,22 \pm 0,06)$; il en est de même des coefficients de rétention d'azote correspondants $(28,9 \pm 1,3$ et $34,1 \pm 1,2)$.

$\mathrm{Au}$ cours des semaines qui suivent l'épilage, les variations sont beaucoup plus fortes. L'azote digestible que les animaux ont journellement à leur disposition, décroît ; mais les données en $2^{\circ}$ et $7^{\circ}$ semaines sont proches, 4,15 et $3,73 \mathrm{~g}$, tandis que celle en $12^{\prime \prime}$ semaine est nettement plus faible, 2,87 g. Malgré l'apport légèrement moindre en $7^{\prime \prime}$ semaine, les bilans $N$ et surtout les $C R$ sont plus élevés en $7^{\circ}$ semaine $(1,36 \pm$ $0,04$ et $36,1 \pm 0,9)$ qu'en $2^{\prime \prime}$ semaine $(1,30 \pm 0,04$ et $31,2 \pm 1,0)$, la différence étant hautement significative pour les CR. En 12" semaine, l'abaissement du rationnement et la présence de 15 p. 100 de cæcotrophes riches en protéines, ont fortement diminué l'apport en $\mathrm{N}$ digestible $2,87 \mathrm{~g} / \mathrm{j}$, d'où un abaissement du bilan $\mathrm{N}$ à $0,79 \mathrm{~g} / \mathrm{j}$. Le coefficient de rétention n'est plus que de $28,6 \pm 0,2$. Les données relatives à la 12 " semaine sont significativement plus faibles que celles des $2^{\prime \prime}$ et $7^{\circ}$ semaines.

Grâce à la formule mise au point par Parigini-BINı \& Cesseli (1977), qui permet de calculer la valeur énergétique de l'urine de lapin à partir de sa concentration en azote : En $(\mathrm{Nu})(\mathrm{Kcal} / 24 \mathrm{~h})=-0,72 \pm 12,37 \mathrm{Nu}$ g nous avons calculé l'énergic métabolisable apportée par la ration au cours de chacun des bilans. Celle-ci est pratiquement constante 2543 à $2609 \mathrm{Kcal} / \mathrm{kg}$ MS. Ces données conduisent à un rendement $\mathrm{EM} / \mathrm{ED}$ de 95 p. 100.

\section{Discussion}

La production de poils collectés en 52 semaines, $983 \pm 31 \mathrm{~g}$ par animal, est très satisfaisante. L'augmentation des refus, toujours assez faible, en fonction de la croissance des poils, et malgré les moindres quantités offertes, indique que le plan alimentaire est très légèrement en deçà des besoins des animaux.

Sur l'ensemble de l'année, nos lapines se séparent en deux groupes : celles dont le poids est stable ou qui maigrissent un peu, et celles qui grossissent. Le surplus d'ingéré des animaux qui consomment la quasi-totalité de leur ration par rapport à ceux qui laissent des refus, semblerait plutôt úilisé pour une augmentation de poids que pour une production de poils. Cette production de poils accompagnant un gain de poids a été observée par McGraham \& Searle (1982) et par Duplessis \& De WET (1983) chez le mouton en croissance.

Il n'est pas observé de différence significative entre les CUD de la matière organique et ceux de l'énergie. ERIKsson (1952) sur lapins adultes, observe la même égalité lorsque la ration qu'il utilise est identique à la nôtre; de même ceux d'EgGum et al. (1982) dès que le poids vif des sujets atteint $2 \mathrm{~kg}$. Ces derniers auteurs observent un rapport EM/ED de 94, pratiquement égal à celui que nous avons mesuré : 95 p. 100.

La présence de cæcotrophes en $12^{\prime \prime}$ semaine explique les faibles coefficients de digestibilité et de rétention d'azote à ce moment. L'abondance de poils pourrait expliquer que l'animal soit gêné dans l'accomplissement de la cæcotrophie. La perturbation de ce phénomène et conjointement l'abaissement du niveau alimentaire 
pourraient être considérés comme responsables des pertes pondérales observées. Elle pourrait peut-être aussi expliquer que la production annuelle de poils obtenue avec un épilage toutes les 13 semaines (91 jours) soit légèrement supérieure à celle obtenue avec un épilage tous les 100 ou 110 jours, méthode qui prolonge la durée de cette phase.

Contrairement à la toison de mouton, le poil Angora est une matière relativement pure (Frolich, 1969). La connaissance de la composition de ce poil (eau : 13,5 p. 100 ; matières grasses et impuretés : 1,5 p. 100 ; protéines : 85 p. 100) permet d'estimer le dépôt protéique de cette production à $835,5 \mathrm{~g}$ par an.

Le gain pondéral durant les 52 semaines d'expérience est de $180 \mathrm{~g}$. Si on admet les données de De Blas \& Galvez (1975), relatives à la composition corporelle du lapin (poids vif vide) en fin de croissance (30 p. 100 MS et 60 MAT p. $100 \mathrm{MS}$ ), cette augmentation de poids correspondrait à un dépôt de $32 \mathrm{~g}$ de protéines. La variation annuelle due aux changements de poids vif représenterait moins de 4 p. 100 du dépôt protéique de poils.

Le calcul d'un tel rapport semaine par semaine est illusoire. En effet, les variations pondérales hebdomadaires sont très fluctuantes d'une semaine à l'autre et d'un animal à l'autre. Si on élimine le premier bilan durant lequel tous les animaux ont maigri, la variation de poids vif enregistrée durant les semaines de bilan a oscillé de -120 à $+110 \mathrm{~g}$. Il semble donc très aléatoire de préjuger d'une modification de la composition corporelle de l'animal à partir des données pondérales recueillies.

Etant donné l'état adulte de nos animaux, il est vraisemblable que cette modification ait porté plus sur les matières grasses que sur les protéines : on observe juste après l'épilation la présence de dépôts gras superficiels importants. Ce n'est que globalement qu'il est possible de dire que le poids «nu» des animaux est croissant en $2^{\circ}$, stationnaire en $7^{\circ}$ et décroissant en $12^{\circ}$ semaine.

L'azote digestible $(\mathrm{Nd})$ que l'animal a à sa disposition lui permet de couvrir ses besoins d'entretien et ceux correspondant à sa production de poils. Nous n'avons pas trouvé de données relatives à l'azote métabolique fécal et endogène urinaire chez le lapin adulte. Mais les résultats d'Eriksson (1952) qui a fait varier de 5,5 à 35,5 p. 100 les teneurs en protéines des rations qu'il distribuait à des lapins adultes d'un poids moyen de $3 \mathrm{~kg}$, montrent que les bilans $\mathrm{N}$ sont pratiquement nuls lorsque ces teneurs sont aux environs de 10 p. 100 ; ce qui permet de calculer un besoin journalier de $0,75 \mathrm{~g}$ de $\mathrm{Nd}$. Pour les nôtres, dont le poids moyen est proche de $4 \mathrm{~kg}$, on peut admettre que ce besoin est de $1 \mathrm{~g} / \mathrm{j}$.

En 364 jours, les lapines ont ingéré en moyenne $49275 \mathrm{~g}$ de matière sèche et ont eu à leur disposition $1083 \mathrm{~g}$ d'Nd dont $364 \mathrm{~g}$ ont été utilisés pour l'entretien. Les $719 \mathrm{~g}$ restant ont été disponibles pour la production : ils ont permis le dépôt de $134 \mathrm{~g}$ sous forme de poil. Le rendement global annuel du dépôt protéique de poil serait apparemment de 18,5 p. 100. Mais ce raisonnement ne tient pas compte de l'appareil folliculaire inclus dans le derme. En effet, le bulbe pileux fournit, en plus du poil, la gaine interne composée d'une scléroprotéine dépourvue de cystine et qui se dissout au niveau du canal pilosebacé pour libérer le poil kératinisé. Cette gaine interne est produite en quantité équivalente à celle du poil mais on ignore dans quelle mesure les produits de digestion sont réabsorbés ou éliminés : il existe donc là une inconnue pour évaluer le rendement des protéines dans la production de poil (Rougeot \& ThÉBAult, 1984). 
La perte de poids observée consécutivement à l'épilage pourrait partiellement être la conséquence du stress de l'épilage lui-même, bien que celui-ci soit effectué rapidement. Il semble plus logique de l'attribuer au choc thermique brutal qui lui est consécutif. L'épilage provoque une modification importante du proche environnement et le comportement des animaux est très démonstratif : decubitus latéral et détente tranquille avant, attitude ramassée et contractée immédiatement après et durant les jours suivants surtout si la température ambiante est basse. L'importance indiscutable de la déperdition énergétique lorsque l'animal est nu et l'ensemble du phénomène de thermo-régulation sont confirmés par l'intensité de la reprise pondérale plus lente en début d'hiver et de printemps qu'en début d'été et d'automne. Il serait intéressant de mesurer l'importance de ces déperditions pour pallier leurs inconvénients.

Inversement, on peut penser que durant les 2 ou 3 semaines qui précèdent l'épilage, la couverture de poils gêne l'animal pour évacuer sa chaleur, phénomène qui expliquerait la limitation de l'ingéré dès la $11^{\mathrm{e}}$ et en $12^{\mathrm{e}}$ semaine surtout au printemps et en été ( $3^{\mathrm{e}}$ semaine de juin et de septembre) chez la majorité des animaux. Une légère suralimentation post-épilage serait favorable aux animaux, un moindre apport avant l'épilage éviterait un gaspillage.

L'étude de l'évolution pondérale que nous avons effectuée et les connaissances sur l'évolution du dépôt $\mathrm{N}$ sous forme de poil suggèrent que pour effectuer une mesure précise du rendement du dépôt $\mathrm{N}$ sous forme de poil, il faudrait éliminer les semaines où les variations pondérales sont fortes, les 3 premières et les 3 dernières semaines du cycle, et dans les semaines 4 à 10 choisir celles où la production est maximale : de la $4^{\circ}$ à la $6^{\circ}$.

\section{Conclusion}

Le peu de refus laissé par les animaux tout au cours de l'année et leur production de poils élevée, proche $\mathrm{du} \mathrm{kg}$ par an, conduisent à penser que le plan alimentaire saisonnier appliqué à l'élevage de lapins Angora de I'I.N.R.A. demande peu de corrections.

Contrairement à notre hypothèse initiale, peut-être par suite des variations assez faibles de la température ambiante $\left(14-24^{\circ} \mathrm{C}\right)$, l'évolution pondérale des lapines Angora est très comparable d'une saison à l'autre : diminution du poids vif «nu» durant les jours qui précèdent et les jours qui suivent l'épilation et reprise lente ultérieurement. Ces diminutions sont plus faibles en début d'été et d'automne qu'en début d'hiver et de printemps. La baisse du niveau alimentaire dès la $10^{e}$ semaine et la régression du comportement de cæcotrophie dès la $11^{\circ}$ expliquent la perte de poids préépilage. Cette régression est sûrement la cause de perturbation chez le lapin Angora et un argument pour épiler toutes les 13 semaines (91 jours) et non tous les 100 jours.

Le stress provoqué par l'épilation et la rupture de l'équilibre thermique qui s'ensuit pourraient expliquer les mortalités observées à ce moment dans les élevages. La connaissance de ces phénomènes devrait permettre de supprimer ces difficultés. 
Nos bilans montrent l'homogénéité des résultats quelles que soient les saisons et font ressortir des différences importantes entre les semaines postépilatoires.

Le dépôt protéique de poils représenterait pour une année entière 18,5 p. 100 de l'N digestible ingéré par l'animal. Les animaux qui laissent le moins de refus sont ceux qui ont tendance à grossir; toutefois ils ne sembleraient pas être plus aptes à fournir davantage de poils.

\author{
Reçu en juillet 1985. \\ Accepté en novembre 1985.
}

\begin{abstract}
Summary
Nutritional status of Angora doe-rabbit during the 4 seasonal hair growth
\end{abstract}

Very few studies have been made till now about Angora rabbit nutrition. This animal is plucked 4 times a year for hair crop (every 13 weeks).

The feeding pattern used in C.N.R.Z. Angora rabbit breeding, constant whatever the season and decreasing before plucking was applied to 9 Angora doe-rabbits during 52 weeks. Liveweight and level of feed intake were controlled every week and during the 2nd, 7th and 12th post-plucking weeks; digestibility coefficients and $\mathrm{N}$ retention were measured.

Liveweight was not or little affected by the season. It was changed by plucking rhythm : decrease in «naked " liveweight during the days before plucking (lower feed intake and incomplete ingestion of soft feces) and the days after (rapid change in thermal surroundings, chiefly in cold season) and then a slow increase (fig. 1 and 2).

In spite of a constant level of feed intake, $\mathrm{N}$ balances and retention coefficients were statistically lower in autumn and higher in winter (tab. 7).

The decreased levels of feed intake between the 2nd, 7th and 12th weeks did not induce changes in energy digestibility. However, during the 12th week, $\mathrm{N}$ digestibility and retention were lower as soft feces, three times richer in $\mathrm{N}$ than hard ones, were mixed with them (tab. 8).

The annual efficiency of hair production could be 18,5 p. 100.

Important changes in thermal environment, induced by plucking, could produce livewcight falls and induce a higher mortality at this time in Angora farms.

Key words : Angora rabbit, season, hair production.

\title{
Références bibliographiques
}

De Blas J.C., Galvez J.F., 1975. A note on the retention of energy and nitrogen in rabbits. Anim. Prod., 21, 345-347.

Demaux G., Gallouin F., Guemon L., Papantonakis C., 1980. Effets de la privation prolongée du comportement de cæcotrophie chez le lapin. Reprod. Nutr. Develop., 20, 1651-1659.

Du Plessis J.J., De WET P.J., 1981. Nitrogen utilization by sheep. I. Nitrogen utilization by weaned lambs of a wool, a wool/mutton and a mutton/wool breed for wool and body protein formation. Agroanimalia, 12, 21-27. 
Eggum B.O., Chwalibog A., Jensen N.E. and Boisen S., 1982. Protein and energy metabolism in growing rabbits fed sodium hydroxide treated straw. Arch. Tierernähr., 32, 539-549.

Eriksson S., 1952. Metabolism of rabbits at different levels of crude fiber and protein. Kungl. Lantbrukshögsk. Ann., 19, 7-108.

Frolich H.G., 1969. Chemisch-physikalische Kennwerk von Tierhaaren. 1. Mitteilung Angora Kanninhaar. Textilindustrie, 71, 39-41.

Mc C. Graham N., Searle T.W., 1982. Energy and nitrogen utilization for body growth in young sheep from two breeds with differing capacities for wool growth. Aust. J. Agric. Res., 33, 607-615.

Parigini-Bini R., Cesselli P., 1977. Estimate of energy excreted in urine by growing rabbits. Riv. Zootec. Vet., 2, 130-137.

Rougeot J., Colin M., Thébault R.G., 1980. Définition des conditions expérimentales pour l'étude des besoins nutritionnels du lapin angora : nature de la litière et présentation du lest alimentaire. Ann. Zootech., 29, 1-11.

Rovgeot J., Thébault R.G., 1977. Formation de trichobézoards chez le lapin angora nourri ad libitum avec un aliment aggloméré. Rec. Méd. Vet., 153, 655-659.

Rougeot J., Thébault R.G., 1983. Variations saisonnières de la composition et de la structure du pelage : exemple du lapin angora. Ann. Zootech., 32, 287-314.

Rougeot J., Thébault R.G., 1984. Le lapin angora, sa toison, son élevage. Le Point Vétérinaire. Maisons-Alfort, France, $182 \mathrm{p}$.

Rovgeot J., Thébault R.G., 1985. Utilisation d'un agent d'épilation pour faciliter la récolte par épilation du poil de lapin Angora. Cuniculture, 64, 214-216.

Scholaut W., Lange K., 1983. Untersuchungen über die Beeinflussung quantitativer Merkmale der Wolleistung beim Angorakaninchen durch Geschlecht, Alter, Fütterungstechnik und Methionin-gehalt des Futters. Züchtungskunde, 55, 69-84. 